\title{
Design of Inset Microstrip Patch Antenna for Wireless Power Transmission at $2.45 \mathrm{GHz}$
}

\author{
Sajina Pradhan ${ }^{1}$, Sun-Kuk Noh ${ }^{2}$, and Dong-You Choi ${ }^{{ }^{*}}$ \\ ${ }^{1}$ Department of Information and Communication Engineering, Chosun University, Gwangju 501-759, Korea \\ ${ }^{2}$ Department of Electronics Engineering, Chosun University, Gwangju 501-749, Korea
}

\begin{abstract}
In communication systems, there are various types of microstrip antenna that can be used for many applications. This paper mainly focuses on the simple design of an inset rectangular microstrip patch antenna to operate at a frequency of $2.45 \mathrm{GHz}$ for rectenna design. The study involves using an high frequency structure simulator to design the antenna dimensions and to determine its performance. This antenna is based on a thickness of $1.6 \mathrm{~mm}$ flame retardant 4 (FR-4) substrate having a dielectric constant of approximately 4.7, an inset feed, and a ground plane. After simulation, the antenna performance characteristics such as its return loss, voltage standing wave ratio, gain, and radiation pattern were obtained and compared with the fabricated measured antenna.
\end{abstract}

Index Terms: Microstrip patch antenna, Inset feed technique, Radiation pattern, Wireless communication

\section{INTRODUCTION}

Wireless communication has been developed rapidly in the past decades and it already has a great impact on human life. In the field of wireless communications, antennas play a vitally important role. In the late 1970s, there was rapid development in the field of microstrip patch antenna technology. In early 1980s, antenna elements and arrays were developed in terms of design and modeling. Due to their advantages like light weight, reduced size, low cost, conformability, and the ease of integration with an active device, these antennas have been extensively studied. A microstrip patch antenna is the combination of a radiating patch on one side of the dielectric substrate and ground on the other side. The patch is generally made of a conducting material like gold or copper. The radiating patch and the feed lines are usually photo etched on the dielectric substrate. Microstrip patch antennas radiate primarily because of the fringing fields between the patch edge and the ground plane. Therefore, the antenna can be fed by a variety of methods. These methods can be classified into two categories: contacting and non-contacting. In the contacting method, the radio frequency (RF) power is fed directly to the radiating patch using a connecting element such as a microstrip line or probe feed. In the noncontracting method, electromagnetic field coupling is performed to transfer power between the microstrip line and the radiating patch which includes proximity feeding and aperture feeding $[1,2]$.

Microstrip antennas are characterized by a large number of physical parameters. They are designed to have many geometrical shapes and dimensions but rectangular and circular microstrip patches have been used in many applications. In this paper, the design of an inset feed rectangular microstrip patch antenna at $2.45 \mathrm{GHz}$ for wireless communication is presented having length $\mathrm{L}$, patch

Received 06 March 2012, Revised 06 April 2012, Accepted 09 April 2012

*Corresponding Author E-mail: dychoi@chosun.ac.kr 
width $\mathrm{W}$, inset fed distance Yo, and transmission line length Lf, as shown in Fig. 1. This antenna is designed on flame retardant 4 (FR-4) substrate and its performance characteristics, which include return loss, voltage standing wave ratio (VSWR), gain, and radiation pattern, were obtained from a simulation.

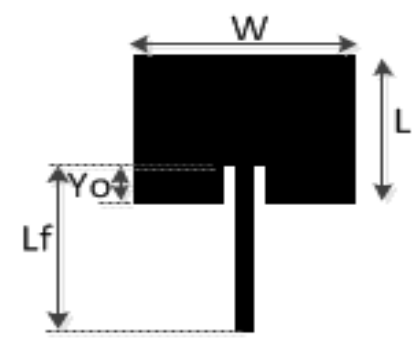

Fig. 1. Inset rectangular microstrip patch antenna.

\section{ANTENNA GEOMETERY AND ITS DESIGN REQUIREMENTS}

For a rectangular patch, the length $\mathrm{L}$ of the patch is usually $0.333 \lambda o$, where $\lambda o$ is the free space wavelength. The patch is selected to be very thin, such that the height of the substrate $\mathrm{h}<<\lambda_{0}$. Thus, a rectangular patch of dimensions $36.26 \times 27.90 \mathrm{~mm}$ is positioned on one side of an FR-4 substrate of thickness $1.6 \mathrm{~mm}$, and the ground plane with dimension of $150 \times 150 \mathrm{~mm}$ is located on the other side of the substrate.

There are three essential parameters for the design of an inset fed rectangular patch antenna. They are resonant frequency $\left(f_{\mathrm{r}}\right)$, dielectric material of the substrate $\left(\varepsilon_{\mathrm{r}}\right)$, and the thickness of the substrate. The resonant frequency selected for this design is $2.45 \mathrm{GHz}$.

The dielectric material of the substrate selected for this design is FR-4, which has a dielectric constant of the 4.7 and a loss tangent equal to 0.019 . The dielectric constant of the substrate material is an important design parameter. A low dielectric constant of the substrate material is used in the prototype design because it gives better efficiency, a higher bandwidth, and a low quality factor $\mathrm{Q}$. The low value of the dielectric constant increases the radiated power. The design has a patch size independent of the dielectric constant. Therefore, the reduction in the patch size is accomplished by using a higher dielectric constant and FR-4 is good in this agreement. The small loss tangent was neglected in the simulation.

The thickness of the substrate is another important design parameter. The thickness of the substrate increases the fringing field at the patch periphery like the low dielectric constant and increases the radiated power. The height of the dielectric substrate of the inset microstrip patch antenna is $h$ $=1.6 \mathrm{~mm}$.

\section{MATHEMATICAL CALCULATION OF THE ANTENNA PARAMETERS}

The parameters of the antenna can be calculated by the transmission line method.

\section{A. Width of the Patch}

The width of the antenna can be determined by

$$
W=\frac{\lambda o}{2}\left(\frac{\varepsilon_{r}+1}{2}\right)^{-\frac{1}{2}}
$$

\section{B. Length of the patch}

The effective constant can be obtained by

$$
\varepsilon_{\text {eff }}=\left(\frac{\varepsilon_{r}+1}{2}\right)+\left(\frac{\varepsilon_{r}-1}{2}\right) \frac{1}{\left(1+\frac{12 h}{W}\right)^{\frac{1}{2}}}
$$

where

$\varepsilon_{\text {eff }}=$ elective dielectric constant

$\varepsilon_{r}=$ dielectric constant of substrate

$h=$ height of dielectric substrate

$W=$ width of the patch

The dimensions of the patch along its length have $n$ ow been extended on each end by a distance $\Delta L$, whic $\mathrm{h}$ is given by

$$
\Delta L=0.412 h \frac{\left(\varepsilon_{\text {eff }}+0.3\right)\left(\frac{W}{h}+0.264\right)}{\left(\varepsilon_{\text {eff }}-0.258\right)\left(\frac{W}{h}+0.8\right)}
$$

The actual length $\mathrm{L}$ of the patch is given by

$$
L=\frac{1}{2 f_{r} \sqrt{\varepsilon_{\text {eff }}}}-2 \Delta L
$$

Where $f_{r}$ is the resonance frequency.

\section{Inset Feed}

The inset feed introduces a physical notch, which in 
turn introduces a junction capacitance. The physical notch and its corresponding junction capacitance influence the resonance frequency. As the inset feed-point moves from the edge toward the center of the patch, the resonant input impedance decreases monotonically and reaches zero at the center. When the value of the inset feed point approaches the center of the patch, $\operatorname{Cos}^{2} \frac{\Pi}{L} y_{0}$ where $y_{0}$ is the inset distance, which varies very rapidly; therefore, the input resistance also changes rapidly with the position of the feed point. To maintain very accurate values, a close tolerance must be preserved [3-5]. In this paper, the value of the inset-fed distance $y_{0}$ is $7.5 \mathrm{~mm}$ and the length and width of the transmission line at $50 \Omega$ are $32 \mathrm{~mm}$ and 3 mm, respectively.

\section{SIMULATION AND MEASUREMENT RESULTS}

The antenna was simulated in high frequency structure simulator (HFSS), which is a high performance full-wave electromagnetic (EM) field simulator for arbitrary 3D volumetric passive devices, with a Microsoft Windows graphical user interface. The antenna designed in the HFSS simulator is shown in Fig. 2.

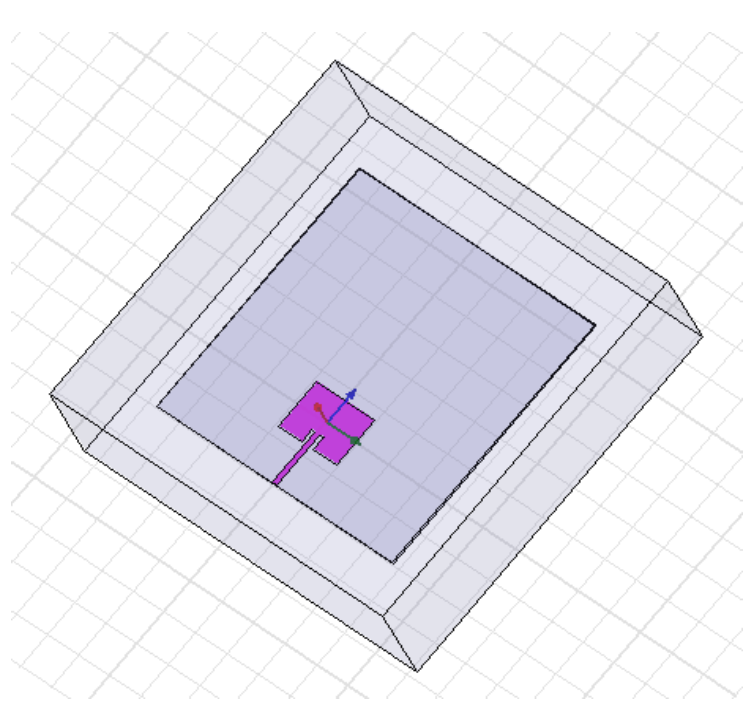

Fig. 2. Antenna in high frequency structure simulator.

The simulated return loss of the inset-fed antenna is $31.07 \mathrm{~dB}$ at the center frequency of $2.45 \mathrm{GHz}$ as shown in Fig. 3. by

The bandwidth of the antenna as a percentage is defined

$$
B W=\frac{f_{\max }-f_{\min }}{f_{r}} * 100 \%
$$

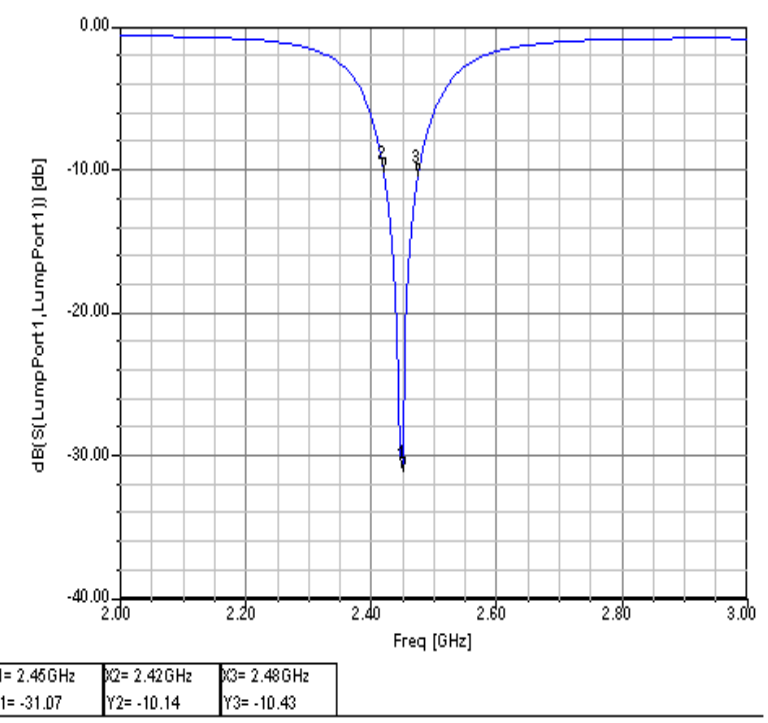

Fig. 3. Return loss vs. frequency.

where $f_{\max }$ and $f_{\min }$ are determined at $-10 \mathrm{~dB} . f_{r}$ is the resonance frequency. The simulated value at $-10 \mathrm{~dB}$, $f_{\text {max }}=2.48, f_{\min }=2.42$, and the bandwidth is $2.44 \%$.

The VSWR characteristic of the antenna should fall in between 1 and 2. From simulation, its value is 1.06 at 2.45 $\mathrm{GHz}$ as depicted in Fig. 4.

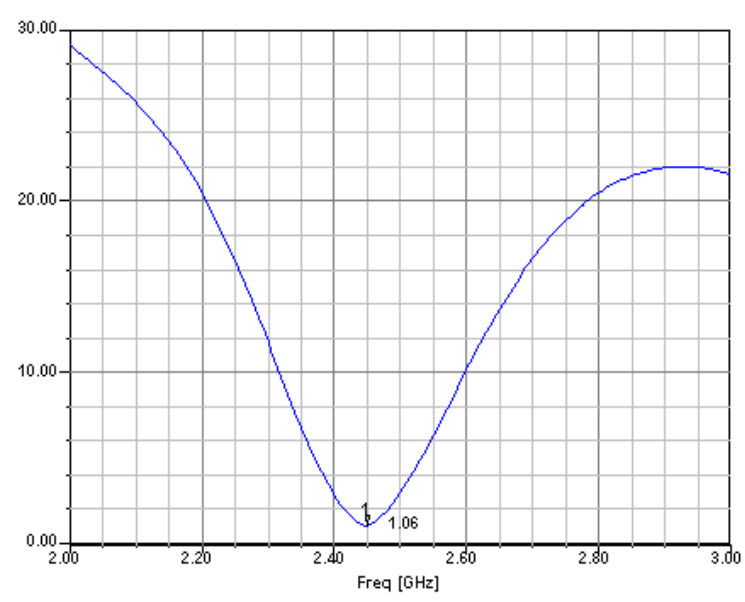

Fig. 4. Voltage standing wave ratio vs. frequency. 
Also, the radiation pattern of the antenna obtained is shown in Fig. 5 at phi $=90$ degrees (red) and phi $=0$ degrees (blue) at $2.45 \mathrm{GHz}$. This shows the gain of the antenna is $3.408 \mathrm{~dB}$.
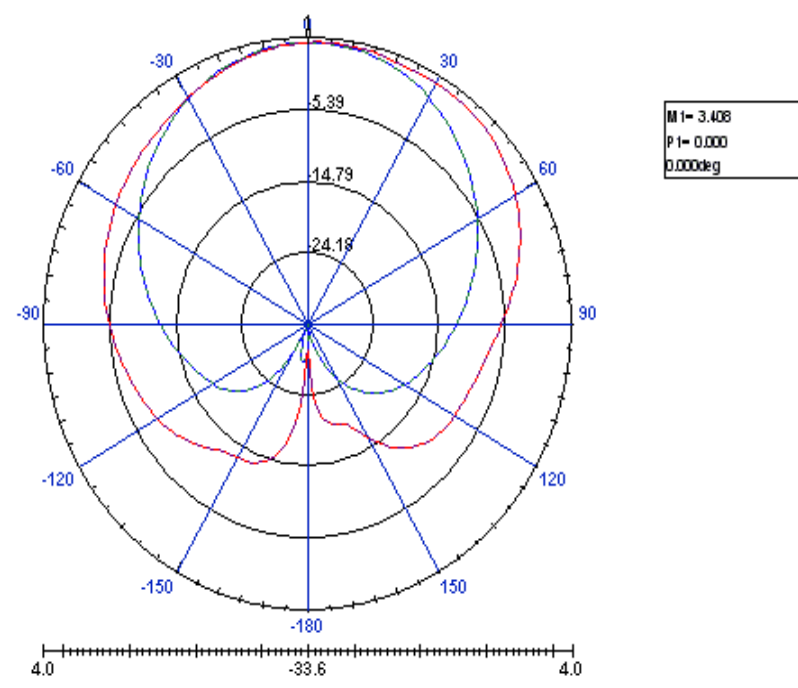
pions

Fig. 5. Radiation pattern at $2.45 \mathrm{GHz}$

The scattering parameter for the antenna design at 2.45 GHz is shown in the Smith chart in Fig. 6.

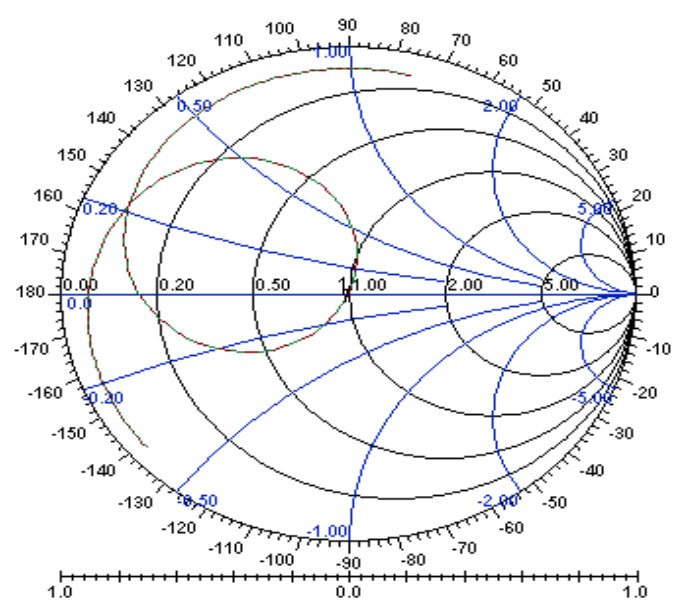

Fig. 6. Smith chart at $2.45 \mathrm{GHz}$.

The inset fed microstrip patch antenna is fabricated on a FR-4 printed circuit board (PCB) and the measurement setup is done by using an Agilent Technologies (Santa Clara, CA, USA) N5230A $10 \mathrm{MHz}-40 \mathrm{GHz}$ PNA-L-Network Analyzer as shown in Fig. 7.

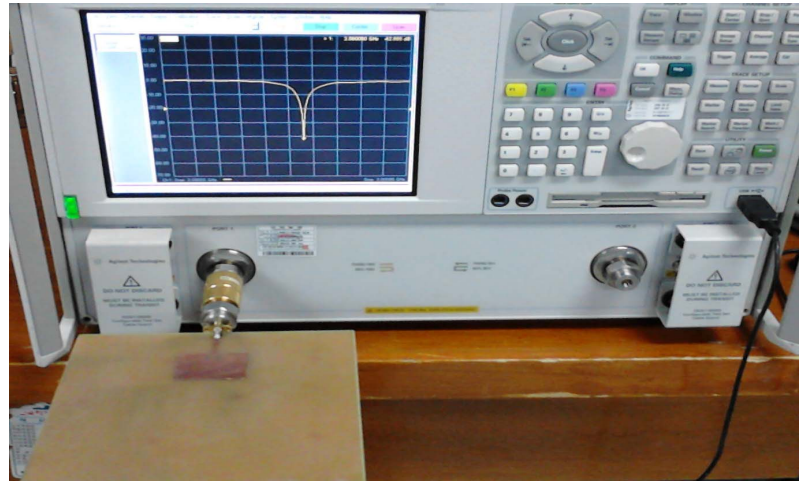

Fig. 7. Measurement setup of inset fed patch antenna.

The measured return loss is obtained to be $-30.551 \mathrm{~dB}$ at $2.56 \mathrm{GHz}$ as shown in Fig. 8 and impedance match at $50 \Omega$ is shown in Fig. 9. The VWSR was measured with an Agilent Technologies N9912A found to be 1.02 at 2.548 $\mathrm{GHz}$ as shown in Fig. 10.

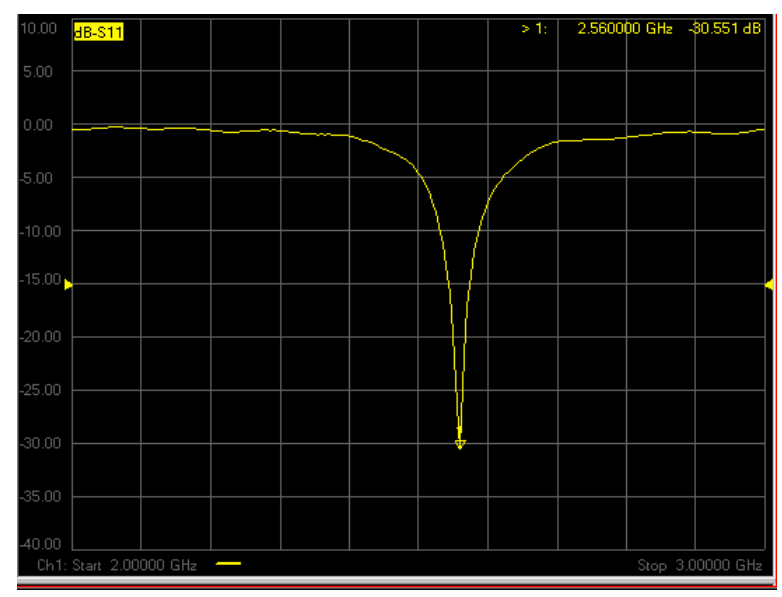

Fig. 8. Measured return loss vs. frequency.

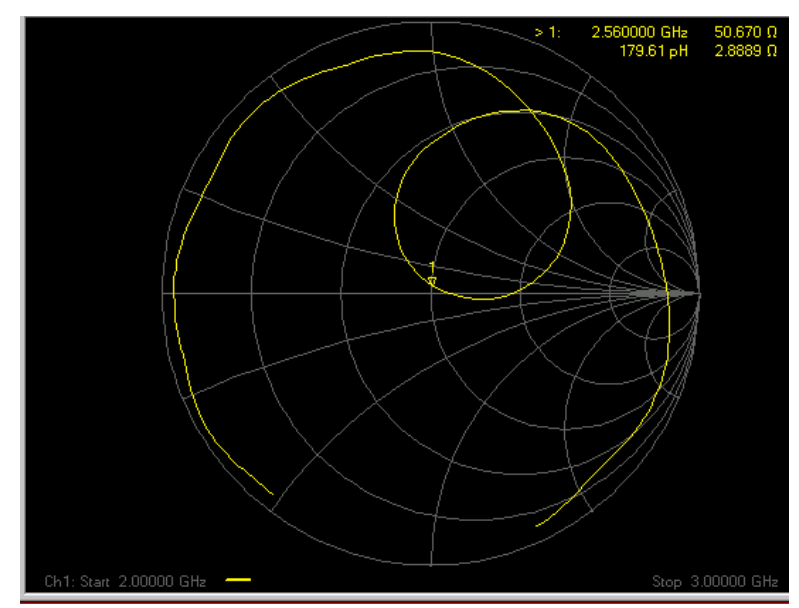

Fig. 9. Impedance matching at $50 \Omega$. 


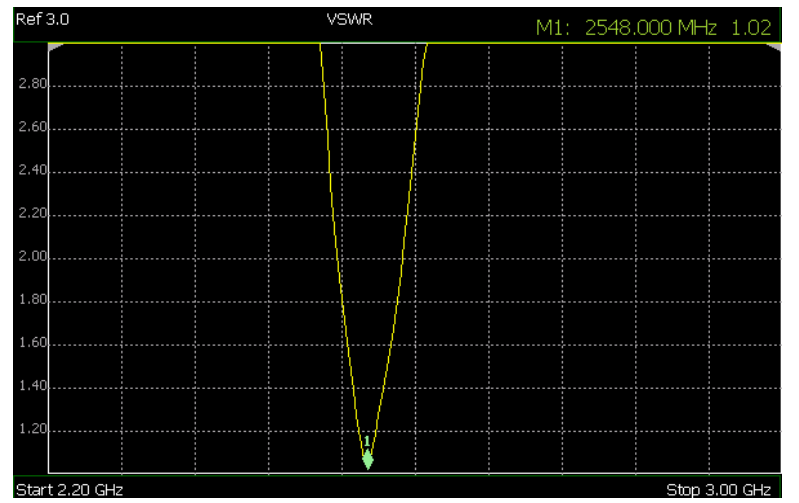

Fig. 10. Measured high frequency structure simulator (VWSR) is found to be 1.02 .

The resonant frequency shifted from the designed frequency of $2.45 \mathrm{GHz}$. The reason for the shift may be due to the FR4 board. In the physical design of the antenna, a material having varying $\varepsilon_{\mathrm{r}}$, length, width, and thickness of the board may cause shifting of the resonant frequency. Other factors may also have an effect, such as the chemical used in etching accuracy and the surface finish. The difference between the simulated and fabricated measured value is shown in Table 1.

Table 1. Comparison between simulation and fabricated measured results

\begin{tabular}{cccc}
\hline Result & $\begin{array}{c}\text { Resonant } \\
\text { frequency (GHz) }\end{array}$ & $\begin{array}{c}\text { Return loss } \\
\text { (dB) }\end{array}$ & VSWR \\
\hline Simulation & 2.45 & -31.07 & 1.06 \\
Measurement & 2.56 & -30.551 & 1.02 \\
\hline
\end{tabular}

VWSR: high frequency structure simulator.

\section{CONCLUSIONS}

In this paper, the mathematical calculation for design of an inset rectangular patch antenna at $2.45 \mathrm{GHz}$ is presented. The simulation of an inset-fed microstrip patch antenna was performed in HFSS. The antenna performance characteristics such as return loss, VSWR, gain, and radiation pattern were obtained in the simulation. The fabricated antenna frequency was compared with the simulated resonance frequency. It was found to be slightly different than the simulated value as real world physical parameters may not be exactly the same.

\section{REFERENCES}

[1] C. A. Balanis, Antenna Theory: Analysis and Design, 2nd ed., New York, NY: John Wiley \& Sons, 1997.

[2] D. M. Pozar, Microwave Engineering, 2nd ed., New York, NY: John Wiley \& Sons, 1998.

[3] Y. Hu, D. R. Jackwon, J. T. Williams, and S. A. Long, "A design approach for inset-fed rectangular microstrip antennas," Proceedings of IEEE Antennas and Propagation Society International Symposium, Albuquerque, NM, pp. 1491-1494, 2006.

[4] M. Ramesh and K. Yip, "Design formula for inset fed microstrip patch antenna," Journal of Microwaves and Optoelectronics, vol. 3, no. 3, pp. 5-10, 2003.

[5] I. Surjati, K. N. Yuli, and A. Astasari, "Microstrip patch antenna fed by inset microstrip line for Radio Frequency Identification (RFID)," Proceedings of Asia-Pacific Symposium on Electromagnetic Compatibility, Beijing, China, pp. 1351-1353, 2010.

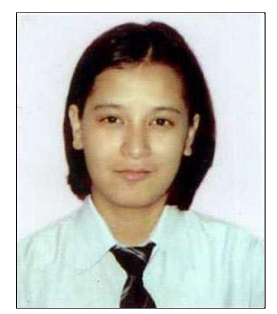

\section{Sajina Pradhan,}

received a B.E. degree in Electronics and Communication Engineering in Nepal in 2007. Since 2010, she has been a Master's student in Communication and Wave Propagation Lab, Department of Information and Communication, Chosun University, Gwangju, South Korea. Her research areas of interest are RF Energy harvesting devices, designs, and applications.

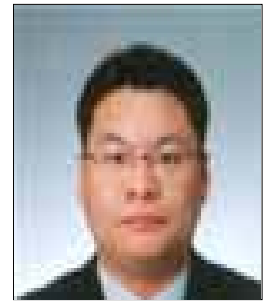

\section{Sun Kuk Noh}

received the B.Sc., the M.Sc., and the D.Sc. degrees in Electronics Engineering from Chosun University of Gwangju, Republic of Korea, in 1995, 1997, and 2000, respectively. From March 2002 to August 2004, he was a Research Professor in the Division of Electronics and Information Engineering, Chonbuk National University, Republic of Korea. Since Sept. 2004, he has been a Full-time Lecturer in the Department of Mobile Communications Engineering, Honam University, Gwangju, Korea. His major research interests include mobile communications, radiowave propagation, DMB, RFID, and USN. He is a Member of IEEE, JCN, IEEK, and KICS. 


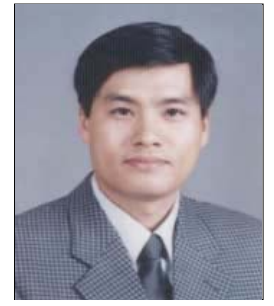

\section{Dong You Choi}

received the B.S., M.S., and Ph.D. degrees in the Department of Electronic Engineering from Chosun University, Gwangju, Korea in 1999, 2001, and 2004, respectively. Since 2007, he has been teaching and researching as an assistant professor at Chosun University. His research interests include mobile communication and wave propagation. He is a member of IEICE, JCN, KEES, IEEK, and KICS. 